\title{
Performance Evaluation of OOK, DPSK and Duo-binary Modulation Format based Mixed-Line-Rate (MLR) Optical Network
}

\author{
Rochak Bajpai, Sujata Sengar, Sridhar Iyer and Shree Prakash Singh
}

\begin{abstract}
With the steady increase in the heterogeneous Internet traffic, the optical wavelength division multiplexed (WDM) networks based on a mixed line rate (MLR) strategy have emerged as an efficient-solution. Also, with the migration from the legacy to the higher line rate(s), the advanced modulation format(s) (MF) is/are required. However, use of appropriate $\mathrm{MF}(\mathrm{s})$ for the higher line rate(s) still remains an open problem. In this article, we compare the performance of an On-Off Keying (OOK), Differential Phase Shift Keying (DPSK) and Duo-binary (DB) MF based MLR network in the presence of various physical layer impairment(s) (PLIs) for which, we propose a mathematical model based on various MFs. As a novelty, we validate the proposed theoretical model's results by comparing them with the results obtained through simulations from OptSim, which has not been conducted in any existing study(s) thus far. Our simulation results show that the DB MF is perfectly suitable for high spectral-efficient MLR systems owing to its high resistance to various PLIs.
\end{abstract}

Keywords-Duo-binary, DPSK, MLR, OOK, $Q$-factor.

\section{INTRODUCTION}

With the continuous advancement in the telecommunication networks, the Internet traffic has grown significantly which has led to a rapid increase in the bandwidth requirement in the long-haul networks. In order to meet the diverse service requirements, the traffic flow in the backbone networks has become increasingly heterogeneous. For satisfying the requirement of the diverse traffic, the concept of mixed line rate (MLR) optical wavelength division multiplexed (WDM) network has evolved in which, the channels are combined as sub-bands, each of which operates at the same bit rate; hence, the channels at various line rates are combined and transmitted over the same fiber [1]. However, in the MLR networks, when the signal traverses for long distances, the effect of the various physical layer impairments (PLIs) become prominent which results in the degradation of the signal quality and hence, an increase in the bit error rate

Manuscript received April 27, 2018, revised August 14, 2018.

Rochak Bajpai, Sujata Sengar, and Shree Prakash Singh are with Division of ECE, Netaji Subhas Institute of Technology, Sector-3, Dwarka, New Delhi-110078. e-mail: rochakbajpai@gmail.com sujata@nsit.com, sps nsit@yahoo.co.uk.

Sridhar Iyer is with Department of ECE, Jain College of Engineering, T S. Nagar Hunchanatti Cross-Machhe, Belagavi, Karnataka, India 590014; e-mail: sridhariyer1983@ gmail.com.
(BER) [2]. The various major PLIs which affect the MLR network performance are: (i) chromatic dispersion (CD), (ii) cross phase modulation (XPM), (iii) four wave mixing (FWM), (iv) accumulated noise (i.e., amplified spontaneous emission (ASE) noise), and (v) inter- and intra-channel interferences [3, 4]. Further, the impact of these PLIs on the performance of a MLR network, in terms of the quality of transmission (QoT), is strongly dependent on the (i) accumulated power, and (ii) individual power of the other optical channels which are transported simultaneously on the same fiber [4].

In the legacy 10 Gbps optical WDM networks, the intensity modulation direct detection (IM/DD) scheme is utilized and since the network is of a fixed grid type, it follows the International Telecommunication Union (ITU-T) defined fixed channel spacing of $50 \mathrm{GHz}$ [4]. Hence, a modulated signal at $10 \mathrm{Gbps}$, using IM/DD, requires a spectrum of less than $15 \mathrm{GHz}$ which implies that, such a system has a spectral efficiency (SE) of $0.2 \mathrm{~b} / \mathrm{s} / \mathrm{Hz}$ which leads to a poor spectrum utilization. Hence, the use of higher line rates such as $40 \mathrm{Gbps}$ is necessitated which, compared to the legacy line rates, under similar conditions, fundamentally decreases the CD tolerance of a system [5]. However, higher line rate(s) systems require advanced modulation formats (MFs) with narrow optical spectrum and also enhance SE [6].

At a line rate of $40 \mathrm{Gbps}$, compared to the IM/DD scheme, owing to its inherent improved receiver sensitivity of $3 \mathrm{db}$, differential phase shift keying (DPSK) is the preferred system MF [7, 8]. Duo-binary (DB) signalling is an alternate MF which provides high tolerance to $\mathrm{CD}$ in the systems whose performance is primarily limited due to dispersion $[9,10]$. Further, the DB format is easy to implement, and has small optical bandwidth which results in more channels being placed close to each other leading to improved SE. Also, the SE can be improved by adopting a gridless network, in which, the channel spacing within the sub-bands and between the different sub-bands is reduced [4]. In addition, with guard band (GB), the channels are separated by an amount equal to the spectrum of its signal, which implies that there occurs a tighter packing of the channels. However, this reduction in inter-channel spacing gives rise to the inter-channel crosstalk which results in channel performance degradation [4].

In this article, we compare the performance of a $40 \mathrm{Gbps}$ 
MLR WDM network based on (i) NRZ-OOK, (ii) DPSK, and (iii) DB MFs, with the variation of power and in the presence of various PLIs. The main aim of this article is to present a rigorous analysis of the performance of OOK, DPSK and DB systems at $40 \mathrm{Gbps}$ in the presence of CD, XPM, FWM and ASE noise. Further, the novelty of our work that distinguishes it from the existing studies is that we (i) mathematically detail the MLR network considering various MFs, and (ii) validate the proposed theoretical model's results by comparing them with those obtained through simulations from OptSim, which to our knowledge has not been done thus far. Our results show that the DB MF based system outperforms the OOK and the DPSK MFs based system.

Since our current study extensively uses many acronyms, for the ease of readability, in Table I, we list the various acronyms used in the study, and their corresponding description.The rest of the article is structured as follows: In Section II, we describe the system model. Section III details the theoretical performance evaluation considering the various modulation formats. Section IV discusses the obtained simulation results. Finally, Section V concludes the study.

Table I List of acronyms used in the study, and their corresponding descriptions.

\begin{tabular}{|c|c|}
\hline Acronym & Description \\
\hline ASE & amplified spontaneous emission \\
\hline BER & bit-error rate \\
\hline CD & duo-binary \\
\hline DB & fifferential phase shift keying \\
\hline DPSK & mixed line rate \\
\hline FWM & on-off keying \\
\hline MLR & physical layer impairments \\
\hline OOK & standard single mode fiber \\
\hline PLI & quality factor \\
\hline SSMF & dispersion compensation \\
\hline Q-factor & cross phase modulation \\
\hline DC & quality of transmission \\
\hline XPM & probability of error \\
\hline QoT & \\
\hline IM/DD & intensity modulation direct detection \\
\hline P & arong \\
\hline
\end{tabular}

\section{II.SYSTEM MODEL}

The MLR WDM link architecture considered in the study is shown in Fig. 1, which emulates a WDM link in a network topology. The MLR WDM link corresponds to eleven users transmitting at different line rates using various MFs. All the signals are then multiplexed and transmitted over a standard single mode fiber (SSMF) of length $L \mathrm{kms}$ with dispersion compensation (DC). The values of the dispersion, the attenuation, the core area, and the nonlinear refractive index of the SSMF are $17 \mathrm{ps} / \mathrm{nm} \mathrm{km}, 0.2 \mathrm{db} / \mathrm{km}, 50 \mu \mathrm{m}^{2}$, and $2.7 \times 10^{-20} \mathrm{~m}^{2} / \mathrm{W}$, respectively. In the WDM link, the center channel transmits at $40 \mathrm{Gbps}$, and the surrounding channels (i.e., five channels on either side of the center channel) transmit at NRZ-OOK 10Gbps. Two MLR cases are considered: Case-1 does not deploy an amplifier i.e., $L=80$ Kms, whereas, Case-2 deploys an amplifier (EDFA) i.e., $L=$

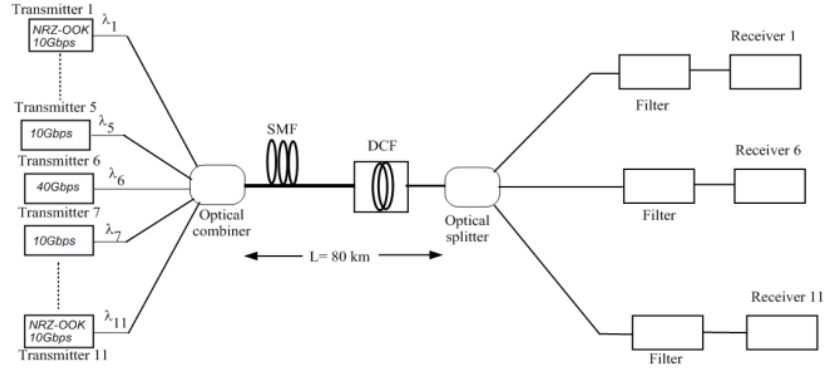

Fig.1. WDM link architecture considered in the study.

$120 \mathrm{Kms}$. Further, in each case, three different configurations are studied in which, the central channel is modulated at 40Gbps using the following MFs: (i) Non-Return-to-Zero (NRZ)-OOK, (ii) NRZ-DPSK, and (iii) NRZ-DB.

\section{PERFormanCE EVALUATION}

In the MLR network, different MFs are used to modulate the signals at various transmission line rates and hence, different performance evaluation models are required. In the following sub-sections, the performance of the central channel is evaluated in terms of the probability of error $\left(P_{e}\right)$ when different MFs are used.

\section{A. Central channel with OOK Modulation}

\section{a. Without Amplifier}

When the central channel at 40Gbps is OOK modulated, then performance of the central channel is worst affected by FWM, and the $P_{e}$ is given as $[3,11]$

$$
P_{e}=\frac{1}{2} \operatorname{erfc}\left[\frac{Q}{(2)^{1 / 2}}\right]
$$

where

$$
Q=\frac{\langle i(1)\rangle-\langle i(0)\rangle}{\sigma(1)+\sigma(0)}
$$

In (2), for the $Q$-factor, the numerator and denominator terms are defined as [11]

$$
\begin{gathered}
\langle i(1)\rangle=R_{0} \mathrm{P}_{\mathrm{S}}^{(\mathrm{r})} \\
\langle i(0)\rangle=2 R_{0}\left[\frac{1}{8} \sum_{k \neq l \neq m} P_{k l m}^{(r)}+\frac{1}{4} \sum_{k=l \neq m} P_{k k m}^{(r)}\right] \\
\sigma^{2}(1)=\sigma_{F W M-\text { signal }^{2}}^{2}(1)+\sigma_{s h}^{2}+\sigma_{T h}^{2} \\
\sigma^{2}(0)=\sigma_{T h}^{2}
\end{gathered}
$$

respectively. In (3) and (4), $R_{0}$ is the responsivity of photodetector, $P_{S}^{(r)}$ the signal power received at receiver, $P_{k l m}^{(r)}$ the FWM component generated on the central channel due to signal on $k^{\text {th }}, l^{\text {th }}$ and $m^{\text {th }}$ channels. In (5) and (6), $\sigma_{s h}^{2}(1), \sigma_{T h}^{2}$ and $\sigma_{F W M}^{2}$-signal represents the variance of 
shot noise, thermal noise, and FWM-signal beat noise, which are given as [11]

$$
\begin{gathered}
\sigma_{s h}^{2}=2 e R_{0} P_{s}^{(r)} B_{e} \\
\sigma_{T h}^{2}=\frac{4 K_{B} T B_{e}}{R_{L}}
\end{gathered}
$$

$\sigma_{F W M-\text { signal }}^{2}(1)=2\left(P_{s}^{(r)}\right)^{2} R_{0}^{2}\left[\frac{1}{8} \sum_{k \neq l \neq m} \frac{P_{k l m}^{(r)}}{P_{s}^{(r)}}+\frac{1}{4} \sum_{k \neq l \neq m=s} \frac{P_{k l s}^{(r)}}{P_{s}^{(r)}}+\frac{1}{4} \sum_{k=l \neq m} \frac{P_{k k m}^{(r)}}{P_{s}^{(r)}}\right]$

respectively. In (8), $R_{L}$ is the receiver load resistance and $B_{e}$ the electrical bandwidth of the receiver which is assumed to be equal to the data rate.

\section{b. With Amplifier}

In this case, an in-line amplifier is deployed which has a uniform gain $G$ over the optical bandwidth $B_{0}$. In addition to amplifying the signal, the amplifier will also generate the ASE noise. When the ASE noise and the signal are incident on a photo detector, the noise beats with itself and also with the signal. As a result the ASE-ASE, ASE-signal and ASEshot noise beat components are generated. In addition, FWMsignal and ASE-FWM beat noise components are also generated. The $P_{e}$ and $Q$-factor are given by (1) and (2), respectively, and in (2), the numerator and denominator terms are defined as [11]

$$
\begin{gathered}
\langle i(1)\rangle=R_{0} P_{s}^{(r)}+R_{0} S_{s p} B_{0} \\
\langle i(0)\rangle=R_{0} S_{s p} B_{0}+2 R_{0}\left[\frac{1}{8} \sum_{k \neq l \neq m} P_{k l m}^{(r)}+\frac{1}{4} \sum_{k=l \neq m} P_{k k m}^{(r)}\right]
\end{gathered}
$$

The noise variances for bit ' 1 ' and ' 0 ' are given by [11] $\sigma^{2}(1)=2 e R_{0}\left[P_{s}^{(r)}+S_{s p} B_{0}\right] B_{e}+4 R_{0}^{2} P_{s}^{(r)} S_{s p} B_{e}+R_{0}^{2} S_{s p}^{2}\left[2 B_{e} B_{0}-B_{e}^{2}\right]+$

$$
\sigma_{F W M-\text { signal }}^{2}(1)+\sigma_{F W M-A S E}^{2}(1)+4 K_{B} T B_{e} / R_{L}
$$

$\sigma^{2}(0)=R_{0}^{2} S_{s p}^{2}\left[2 B_{e} B_{0}-B_{e}^{2}\right]+2 e R_{0} S_{s p} B_{0} B_{e}+\sigma_{F W M-A S E}^{2}(0)+4 K_{B} T B_{e} / R_{L}$

respectively. In (12) and (13), $B_{0}$ is the optical bandwidth of the filter used to limit the ASE noise, $B_{e}$ the ideal electrical filter bandwidth in the receiver, and $S_{s p}$ represents the power spectral density (PSD) of ASE noise, given by

$$
S_{s p}=n_{s p}(G-1) h v
$$

where $n_{s p}$ is spontaneous emission factor ranging from 1.4 to 4 for EDFA. In (12), $\sigma_{F W M-s i g n a l}^{2}(1)$ and $\sigma_{F W M-A S E}^{2}(1)$ are given as [11]

$$
\begin{gathered}
\sigma_{F W M-s i g n a l}^{2}(1)=2\left(P_{s}^{(r)}\right)^{2} R_{0}^{2}\left[\frac{1}{8} \sum_{k \neq l \neq m} \frac{P_{k l m}^{(r)}}{P_{s}^{(r)}}+\frac{1}{4} \sum_{k \neq l \neq m=s} \frac{P_{k l s}^{(r)}}{P_{s}^{(r)}}+\frac{1}{4} \sum_{k=l \neq m} \frac{P_{k k m}^{(r)}}{P_{s}^{(r)}}\right] \\
\sigma_{F W M-A S E}^{2}(1)=4 R_{0}^{2}\left\{2 P_{s}^{(r)}\left[\frac{1}{8} \sum_{k \neq l \neq m} \frac{P_{k l m}^{(r)}}{P_{s}^{(r)}}+\frac{1}{4} \sum_{k \neq l \neq m=s} \frac{P_{k l s}^{(r)}}{P_{s}^{(r)}}+\frac{1}{4} \sum_{k=l \neq m} \frac{P_{k k m}^{(r)}}{P_{s}^{(r)}}\right]\right\} S_{s p} B_{e}
\end{gathered}
$$

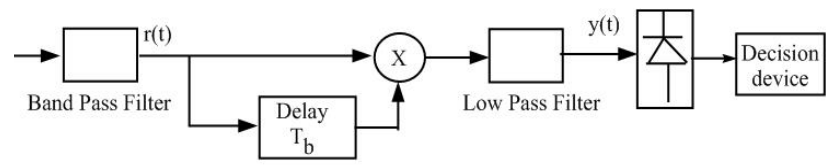

Fig. 2. DPSK demodulator.

respectively. In (13), $\sigma_{F W M-A S E}^{2}(0)$ is given as [6]

$\sigma_{F W M-A S E}^{2}(0)=4 R_{0}^{2}\left\{2\left[\frac{1}{8} \sum_{k \neq l \neq m} P_{k l m}^{(r)}+\frac{1}{4} \sum_{k=l \neq m} P_{k k m}^{(r)}\right]\right\} S_{s p} B_{e}$

\section{B. Central channel with DPSK Modulation}

\section{a. Without Amplifier}

When the central channel at 40Gbps is modulated using DPSK, it is highly susceptible to the XPM induced by the neighbouring OOK channels. Also, XPM affects the 40Gbps channel only when bit ' 1 ' is transmitted over the neighbouring OOK channel. The DPSK modulated signal is detected using a standard delay demodulator followed by a low pass filter, as shown in Fig. 2.

Let the received signal at frequency $f_{s}$ be represented as

$$
r(t)=\sqrt{P_{s}^{(r)}} \cos \left(2 \pi f_{s} t+b_{k} \pi\right)
$$

where $b_{k}=0$ or 1 for $k T_{b}<t<(k+1) T_{b}$ depending on bit ' 0 ' or ' 1 '. In our analysis we consider the worst case scenario in which, bit ' 1 ' is transmitted on all the neighboring OOK channels and bit ' 0 ' is transmitted on the central channel using DPSK modulation with a delayed bit ' 1 '. As symbol ' 1 ' is transmitted on the co-propagating $10 \mathrm{Gbps}$ signal, it generates XPM on the central 40Gbps channel. If bit ' 0 ' is transmitted, then the received signal is expressed as

$$
r(t)=\sqrt{P_{s}^{(r)}} \cos \left(2 \pi f_{s} t\right)+n(t)
$$

where $P_{s}^{(r)}$ represents the signal power at the receiver and $n(t)$ is due to thermal, shot and XPM noise owing to bit ' 1 ' on the neighboring OOK channel. The band pass noise is given as

$$
n(t)=n_{I}(t) \operatorname{Cos}\left(2 \pi f_{s} t\right)-n_{Q}(t) \operatorname{Sin}\left(2 \pi f_{s} t\right)
$$

Hence, the received signal is given as

$r(t)=\sqrt{P_{s}^{(r)}} \cos \left(2 \pi f_{s} t\right)+n_{I}(t) \operatorname{Cos}\left(2 \pi f_{s} t\right)-n_{Q}(t) \operatorname{Sin}\left(2 \pi f_{s} t\right)$

If the delayed bit is bit ' 1 ', then the delayed signal $r(t-T)$ is given as

$$
\begin{aligned}
r\left(t-T_{b}\right)= & \sqrt{P_{s}^{(r)}} \cos \left(2 \pi f_{s}\left(t-T_{b}\right)+1 . \pi\right)+n_{I}\left(t-T_{b}\right) \operatorname{Cos}\left(2 \pi f_{s} t\right)- \\
& n_{Q}\left(t-T_{b}\right) \operatorname{Sin}\left(2 \pi f_{s} t\right)
\end{aligned}
$$

or

$$
\begin{gathered}
r\left(t-T_{b}\right)=-\sqrt{P_{s}^{(r)}} \cos \left(2 \pi f_{s} t\right)+n_{I}\left(t-T_{b}\right) \operatorname{Cos}\left(2 \pi f_{s} t\right)- \\
n_{Q}\left(t-T_{b}\right) \operatorname{Sin}\left(2 \pi f_{s} t\right)
\end{gathered}
$$

In (22) it is assumed that $2 \pi f_{s} T_{b}=2 \pi N$, and hence, (22) can also be represented as

$$
\begin{aligned}
r\left(t-T_{b}\right)= & {\left[\sqrt{P_{s}^{(r)}}+n_{I}\left(t-T_{b}\right)\right] \cos \left(2 \pi f_{s} t\right)-} \\
& n_{Q}\left(t-T_{b}\right) \operatorname{Sin}\left(2 \pi f_{s} t\right)
\end{aligned}
$$


Therefore,

$$
\begin{aligned}
& r(t) r(t-T)=\left\{\left[\sqrt{P_{s}^{(r)}}+n_{X P M_{i}}(t)\right] \operatorname{Cos}\left(2 \pi f_{s} t\right)-n_{X P M_{Q}}(t) \operatorname{Sin}\left(2 \pi f_{s} t\right\} .\right. \\
& \left\{\left[\sqrt{P_{s}^{(r)}}+n_{X P M_{I}}\left(t-T_{b}\right)\right] \cos \left(2 \pi f_{s} t\right)-n_{X P M_{Q}}\left(t-T_{b}\right) \operatorname{Sin}\left(2 \pi f_{s} t\right)\right\} \\
& =\left[\sqrt{P_{s}^{(r)}}+n_{X P M_{1}}(t)\right]\left[P_{s}^{(r)}+n_{X P M_{1}}(t-T)\right] \operatorname{Cos}^{2}\left(2 \pi f_{s} t\right)- \\
& n_{X P M_{Q}}(t)\left[\sqrt{P_{s}^{(r)}}+n_{X P M_{Q}}(t-T)\right] \operatorname{Sin}\left(2 \pi f_{s} t\right) \cdot \operatorname{Cos}\left(2 \pi f_{s} t\right)- \\
& n_{X P M_{Q}}(t-T)\left[\sqrt{P_{s}^{(r)}}+n_{X P M_{t}}(t)\right] \operatorname{Cos}\left(2 \pi f_{s} t\right) \operatorname{Sin}\left(2 \pi f_{s} t\right)+ \\
& n_{X P M_{Q}}(t) n_{X P M_{Q}}(t-T) \operatorname{Sin}^{2}\left(2 \pi f_{s} t\right)
\end{aligned}
$$

The corresponding low pass filtered signal is given as

$$
y(t)=\frac{1}{2}\left[\sqrt{P_{s}^{(r)}}+n_{i}(t)\right]\left[-\sqrt{P_{s}^{(r)}}+n_{i}\left(t-T_{b}\right)\right]+\frac{1}{2} n_{q}(t) n_{q}\left(t-T_{b}\right)
$$

or

$$
\begin{aligned}
y(t)= & \frac{1}{2}\left[-P_{s}^{(r)}+\sqrt{P_{s}^{(r)}} n_{I}\left(t-T_{b}\right)-\sqrt{P_{s}^{(r)}} n_{I}(t)+n_{I}(t) n_{I}\left(t-T_{b}\right)+\right. \\
& \left.n_{Q}(t) n_{Q}\left(t-T_{b}\right)\right]
\end{aligned}
$$

Assuming a high signal to noise ratio (SNR) condition, and neglecting the terms $\left\{n_{I}(t) n_{I}\left(t-T_{b}\right)+n_{Q}(t) n_{Q}\left(t-T_{b}\right)\right\}$ results in

$$
y(t)=\frac{1}{2}\left[-P_{s}^{(r)}+\sqrt{P_{s}^{(r)}} n_{I}\left(t-T_{b}\right)-\sqrt{P_{s}^{(r)}} n_{I}(t)\right]
$$

In (27), $n_{I}\left(t-T_{b}\right)$ and $n_{I}(t)$ are Gaussian with zero mean and variance $\sigma_{n}^{2}$; the term $\sqrt{P_{s}^{(r)}} n_{I}\left(t-T_{b}\right)-\sqrt{P_{s}^{(r)}} n_{I}(t)$ will be Gaussian with zero mean and variance $2 P_{s}^{(r)} \sigma_{n}^{2}$. Under the Gaussian approximation, $P_{e}$ can be expressed as

$$
P_{e}=P(0) P(1 / 0)+P(1) P(0 / 1)
$$

where $\quad P(1 / 0)=\int_{0}^{\infty} \frac{1}{\sqrt{4 \pi P_{s}^{(r)} \sigma_{n}^{2}}} \exp \left(-\frac{\left(x+P_{s}^{(r)}\right)^{2}}{4 P_{s}^{(r)} \sigma_{n}^{2}}\right) d x$ and $P(0 / 1)=\int_{-\infty}^{0} \frac{1}{\sqrt{4 \pi P_{s}^{(r)} \sigma_{n}^{2}}} \exp \left(\frac{\left(x-P_{s}^{(r)}\right)^{2}}{4 P_{s}^{(r)} \sigma_{n}^{2}}\right) d x$ respectively.

The average $P_{e}$ can be expressed as [17]

$$
P_{e}=\frac{1}{2} \operatorname{erfc}\left(\frac{\sqrt{P_{s}^{(r)}}}{2 \sigma_{n}}\right)=\frac{1}{2} \operatorname{erfc}\left(\frac{Q}{\sqrt{2}}\right)
$$

where

$$
Q=\frac{\sqrt{P_{s}^{(r)}}}{\sigma_{n} \sqrt{2}}
$$

In (30), $\sigma_{n}^{2}$ represents the sum of the noise variances due to shot, thermal and XPM noise. The shot and the thermal noise variances are given by (7) and (8), respectively. The XPM noise variance is given as

$$
\sigma_{X P M}^{2}=\frac{P_{t}^{2} \gamma^{2}}{\alpha \sum_{i \neq j} d_{i j}}\left(1+e^{-2 \alpha L}\right)
$$

\section{b. With Amplifier}

With the introduction of an in-line amplifier, ASE noise is also generated. In our analysis we consider the same case as discussed in the DPSK without amplifier i.e., bit ' 0 ' is transmitted on the central channel using DPSK modulation with a delayed bit ' 1 ', and bits ' 1 ' are transmitted on the neighboring OOK channels. The received signal is given as

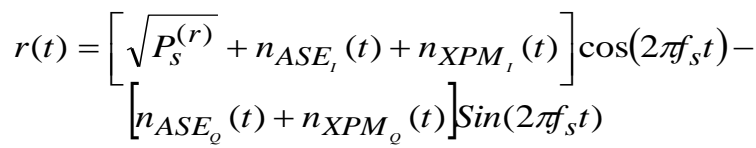

The output of the low pass filter will be [17]

$$
y(t)=r(t) r\left(t-T_{b}\right)
$$

or

$$
\begin{aligned}
y(t)= & \frac{1}{2}\left[\sqrt{P_{S}^{(r)}}+n_{A S E_{l}}(t)+n_{X P M_{l}}(t)\right]\left[-\sqrt{P_{s}^{(r)}}+n_{A S E_{l}}\left(t-T_{b}\right)+n_{X P M_{l}}\left(t-T_{b}\right)\right]+ \\
& \frac{1}{2}\left[n_{A S E_{\varrho}}(t)+n_{X P M_{Q}}(t)\right]\left[n_{A S E_{Q}}\left(t-T_{b}\right)+n_{X P M_{Q}}\left(t-T_{b}\right)\right]
\end{aligned}
$$

or

$y(t)=\frac{1}{2}\left[-P_{s}^{(r)}+n_{A S E_{I}}\left(t-T_{b}\right) \sqrt{P_{s}^{(r)}}+n_{X P M_{I}}\left(t-T_{b}\right) \sqrt{P_{s}^{(r)}}-n_{A S E_{I}}(t) \sqrt{P_{s}^{(r)}}+\right.$ $n_{A S E_{I}}(t) n_{A S E_{I}}\left(t-T_{b}\right)+n_{A S E_{I}}(t) n_{X P M_{I}}\left(t-T_{b}\right)-n_{X P M_{I}}(t) \sqrt{P_{s}^{(r)}}+$

$\left.n_{X P M_{I}}(t) n_{A S E_{I}}\left(t-T_{b}\right)+n_{X P M_{I}}(t) n_{X P M_{I}}\left(t-T_{b}\right)\right]+$

$\frac{1}{2}\left[n_{A S E_{Q}}(t) n_{A S E_{Q}}\left(t-T_{b}\right)+n_{A S E_{Q}}(t) n_{X P M_{Q}}\left(t-T_{b}\right)+\right.$

$\left.n_{X P M_{Q}}(t) n_{A S E_{Q}}\left(t-T_{b}\right)+n_{X P M_{Q}}(t) n_{X P M_{Q}}\left(t-T_{b}\right)\right]$

In (35), under the high SNR condition, $n_{A S E_{I}}(t) n_{A S E_{I}}\left(t-T_{b}\right)$,

$n_{X P M_{I}}(t) n_{X P M_{I}}\left(t-T_{b}\right), n_{A S E_{Q}}(t) n_{A S E_{Q}}\left(t-T_{b}\right)$ and $n_{X P M_{Q}}(t) n_{X P M_{Q}}\left(t-T_{b}\right)$ can be neglected, which results in [17]

$$
\begin{aligned}
& y(t)=\frac{1}{2}\left[-P_{s}^{(r)}+n_{A S E_{I}}\left(t-T_{b}\right) \sqrt{P_{s}^{(r)}}+n_{X P M_{I}}\left(t-T_{b}\right) \sqrt{P_{s}^{(r)}}-\right. \\
& n_{A S E_{I}}(t) \sqrt{P_{S}^{(r)}}+n_{A S E_{I}}(t) n_{X P M_{I}}\left(t-T_{b}\right)-n_{X P M_{I}}(t) \sqrt{P_{S}^{(r)}}+ \\
& n_{X P M_{I}}(t) n_{A S E_{I}}\left(t-T_{b}\right)+n_{A S E_{Q}}(t) n_{X P M_{Q}}\left(t-T_{b}\right)+ \\
& \left.n_{X P M_{Q}}(t) n_{A S E_{Q}}\left(t-T_{b}\right)\right]
\end{aligned}
$$

The various beating terms are a function of the random phase which average out to zero i.e. the means of each beating term will be zero; whereas the variance for each can be expressed as 


$$
\begin{aligned}
& \sigma_{s i g-A S E, I}^{2}=R_{0}^{2} P_{s}^{(r)} S_{s p, I} \Delta f \\
& \sigma_{s i g-X P M, I}^{2}=R_{0}^{2} P_{s}^{(r)} P_{X P M, I} \Delta f \\
& \sigma_{A S E, I-X P M, I}^{2}=R_{0}^{2} S_{s p, I} P_{X P M, I} B_{0} \Delta f \\
& \sigma_{s i g-X P M, I}^{2}=R_{0}^{2} P_{s}^{(r)} P_{X P M, I} \Delta f \\
& \sigma_{A S E, Q-X P M, Q}^{2}=R_{0}^{2} P_{X P M, Q} S_{s p, Q} B_{0} \Delta f
\end{aligned}
$$

where $R_{0}, P_{s}^{(r)}, S_{s p, I}, P_{X P M, I}, S_{s p, Q}, P_{X P M, Q}, B_{0}, \Delta f$ are the responsivity of photo-detector, received signal power, Inphase component of PSD of ASE noise, In-phase component of the XPM power component, Quadrature-phase component of the PSD of ASE noise, Quadrature-phase component of the XPM power component, Optical and Electrical filter bandwidth, respectively. The PSD of ASE noise is given as

$$
S_{s p}=(G-1) n_{s p} h v
$$

Power of the XPM component is given as [12, 13]

$$
P_{X P M}=-2 \gamma<P_{s}>\sum_{p \neq s} P_{p}(o, \omega) H_{s p}^{I M}(\omega)
$$

where $<P_{s}>, P_{p}(o, \omega), H_{s p}^{I M}(\omega)$ are time averaged signal power of the reference channel, PSD of the co-propagating signal $p$, and IM/XPM/IM filter respectively. Further, $H_{s p}^{I M}(\omega)$ is given as

$$
H_{s p}^{I M}(\omega)=\sum_{k=1}^{M} C_{p}^{(k)} e^{\left(j \omega \sum_{i=1}^{k-1} d_{s p}^{i} l_{i}\right)} H_{s p}^{I M(k)}(\omega)
$$

or

$$
H_{s p}^{I M(k)}(\omega)=j\left\{\begin{array}{l}
\exp \left(j \frac{\lambda^{2}}{4 \pi c}\left(l_{k} D_{k}+\hat{D}_{R}^{(k)}\right) \omega^{2}\left(\frac{1-e^{\left(-\alpha_{k}+j\left(d_{s p}^{(k)} \omega-\frac{\lambda^{2}}{4 \pi c} D_{k} \omega^{2}\right)\right) l_{k}}}{\alpha_{k}-j\left(d_{s p}^{(k)} \omega-\frac{\lambda^{2}}{4 \pi c} D_{k} \omega^{2}\right.}\right)\right) \\
\exp \left(-j \frac{\lambda^{2}}{4 \pi c}\left(l_{k} D_{k}+\hat{D}_{R}^{(k)}\right) \omega^{2}\left(\frac{1-e^{\left(-\alpha_{k}+j\left(d_{s p}^{(k)} \omega+\frac{\lambda^{2}}{4 \pi c} D_{k} \omega^{2}\right)\right) l_{k}}}{\alpha_{k}-j\left(d_{s p}^{(k)} \omega+\frac{\lambda^{2}}{4 \pi c} D_{k} \omega^{2}\right)}\right)\right)
\end{array}\right\}
$$

The $P_{e}$ under the Gaussian approximation is given as [17]

$$
P_{e}=\frac{1}{2} \operatorname{erfc}\left(\frac{Q}{\sqrt{2}}\right)
$$

where

$$
Q=\frac{\sqrt{P_{s}^{(r)}}}{\sigma_{n} \sqrt{2}}
$$

and

$$
\begin{aligned}
& \sigma_{n}^{2}=\sigma_{s h}^{2}+\sigma_{t h}^{2}+\sigma_{s i g-A S E, I}^{2}+\sigma_{s i g-X P M, I}^{2}+\sigma_{s i g-A S E, I}^{2}+\sigma_{A S E, I-X P M, I}^{2} \\
& +\sigma_{s i g-X P M, I}^{2}+\sigma_{X P M, I-A S E, I}^{2}+\sigma_{A S E, Q-X P M, Q}^{2}+\sigma_{X P M, Q-A S E, Q}^{2}
\end{aligned}
$$

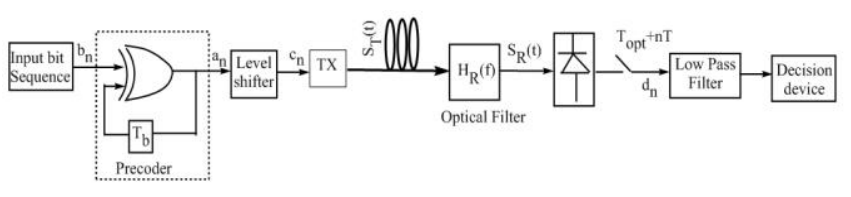

Fig.3. Schematic of the Duo-binary system.

\section{Central channel with Duo-Binary Modulation}

\section{a. Without Amplifier}

In DB modulation, pulses are affected by crosstalk, however, in controlled limits [10]. As shown in Fig. 3, on the transmitter side, the input bit sequence $b_{n}$ is passed through a pre-coder (to avoid error propagation) to generate an output data $a_{n}=b_{n} \oplus a_{n-1}$. The pre-coder output enters a level shifter to generate $c_{n}=2 a_{n}-1$. Assuming that the input bit sequence is $b_{n} \in\{0,1\}$, it follows that $a_{n} \in\{0,1\}$. Now, $c_{n} \in\{-1,1\}$ and the transmitted signal $S_{T}(t)$ at a carrier frequency $f_{s}$ is given as

$$
S_{T}(t)=\left[\sqrt{P_{s}} \sum_{n} c_{n} u(t-n T)\right] e^{j 2 \pi f_{s} t}
$$

The received signal is passed through optical filter. The transfer function of the optical filter is given as

$$
H_{R}(f)=T\left[1+e^{-2 j \pi f T}\right]=2 T \cos \pi f T e^{-j \pi f T} \quad-\frac{1}{2 T} \leq f \leq \frac{1}{2 T}
$$

The receiver is a quadratic receiver using a standard direct detection technique. It is assumed that noises affecting the performance are shot, thermal and FWM noise. The signal at output of the receiver filter is assumed to satisfy DB pulse constraint $x(0)=x(T) \neq 0$ and $x(n T)=0 \forall n \neq 0,1$ , and hence, the received optical signal at an optimum sampling instant is given as

$$
S_{R}\left(t_{o p t}\right)=\left\{\sqrt{P_{s}^{(r)}} d_{n} x(0)+\sqrt{P_{k l m}^{(r)}} e^{-j \theta_{k l m}}\right\}
$$

where $P_{s}^{(r)}$ is the average power of the received signal at frequency $f_{s} \cdot P_{k l m}^{(r)}$ and $\theta_{k l m}$ are the average power and phase of the FWM components generated by the $k^{\text {th }}, l^{\text {th }}$ and $m^{\text {th }} \quad$ channel satisfying $k+l-m=s$ Also, $d_{n}=c_{n}+c_{n-1}+n_{n}$ where $c_{n}$ is the transmitted sequence of amplitudes and $n_{n}$ is a sequence of independent Gaussian random variables which in our case is assumed due to FWM noise. If we assume $c_{n}= \pm 1$ with equal probability, then

$$
d_{n}=\left\{\begin{array}{rrl}
0 & \text { if } & c_{n} \neq c_{n-1} \\
2 & \text { if } & c_{n}=c_{n-1}=+1 \\
-2 & \text { if } & c_{n}=c_{n-1}=-1
\end{array}\right.
$$

Also, when the sample at the output of the optical filter $d_{n}$ is 2 or -2 , it corresponds to the bit sequence $b_{n}=1$ whereas, $d_{n}=0$ corresponds to $b_{n}=0$. The optical signal is detected 
using a photo-detector, whose expression at optimum sampling instant is given as

$$
\begin{aligned}
i_{D}\left(t_{\text {opt }}\right)= & R_{o}\left[\sqrt{P_{s}^{(r)}} d_{n} x(0)+\sqrt{P_{k l m}^{(r)}} \cos \left(\theta_{k l m}\right)\right]^{2}+n_{T h}\left(t_{\text {opt }}\right)+ \\
& n_{S h}\left(t_{\text {opt }}\right)
\end{aligned}
$$

Assuming $P_{s}^{(r)}>>P_{k l m}^{(r)}$ and neglecting $P_{k l m}^{(r)}$ in comparison to $P_{s}^{(r)}$ results in

$$
\begin{aligned}
i_{D}\left(t_{\text {opt }}\right)=R_{o}\left[P_{S}^{(r)} d_{n}[x(0)]^{2}+2 x(0) \sqrt{P_{s}^{(r)} P_{k l m}^{(r)}} \cos \left(\theta_{k l m}\right)\right]+n_{T h}\left(t_{\text {opt }}\right)+ \\
n_{S h}\left(t_{\text {opt }}\right)
\end{aligned}
$$

or

$$
\begin{aligned}
i_{D}\left(t_{\text {opt }}\right)= & R_{o} P_{s}^{(r)} d_{n}[x(0)]^{2}+n_{F W M-s i g n a l}\left(t_{\text {opt }}\right)+n_{\text {Th }}\left(t_{\text {opt }}\right)+ \\
& n_{S h}\left(t_{\text {opt }}\right)
\end{aligned}
$$

where $n_{F W M-\text { signal }}(t)$ represents the FWM-signal beat noise. In (49), apart from FWM-signal beat noise, there will also be thermal and shot noise represented as $n_{T h}(t)$ and $n_{S h}(t)$.

Now, the bit-by-bit decision is employed and the $P_{e}$ can be calculated as

$$
\begin{gathered}
P_{e}=P\left(\frac{\left|i_{D}\left(t_{\text {opt }}\right)\right|>R_{0} P_{s}^{(r)}[x(0)]^{2}}{d_{n}=0}\right) \\
P_{e}==\frac{2}{\sqrt{2 \pi \sigma_{n}^{2}}} \int_{R_{0}} P_{s}^{(r)}[x(0)]^{2} \exp \left[-i^{2} / 2 \sigma_{n}^{2}\right] d s \\
P_{e}=\operatorname{erfc}\left(\frac{R_{0} P_{s}^{(r)}[x(0)]^{2}}{\sqrt{2 \sigma_{n}^{2}}}\right)
\end{gathered}
$$

or

$$
P_{e}=\operatorname{erfc}\left(\frac{Q}{\sqrt{2}}\right)
$$

where

$$
Q=\frac{R_{0} P_{s}^{(r)}[x(0)]^{2}}{\sigma_{n}}
$$

In (52), $\sigma_{n}^{2}=\sigma_{s h}^{2}+\sigma_{T h}^{2}+\sigma_{F W M-s i g n a l}^{2}$, and thermal and shot noise variances are as given in (7) and (8), and signalFWM variance (i.e., $\sigma_{F W M-\text { signal }}^{2}$ ) is given as $[11,13,14]$

$$
\begin{aligned}
\sigma_{F W M-s i g n a l}^{2} & =2\left(P_{s}^{(r)}\right)^{2}[x(0)]^{2} R_{0}^{2}\left[\frac{1}{8} \sum_{k \neq l \neq m} \frac{P_{k l m}^{(r)}}{P_{s}^{(r)}}+\frac{1}{4} \sum_{k \neq l \neq m=s} \frac{P_{k l s}^{(r)}}{P_{s}^{(r)}}+\frac{1}{4} \sum_{k=l \neq m} \frac{P_{k k m}^{(r)}}{P_{s}^{(r)}}\right] \int_{-\frac{1}{2 T}}^{\frac{1}{2 T}}\left|H_{R}(f)\right|^{2} d f \\
& =2\left(P_{s}^{(r)}\right)^{2}[x(0)]^{2} R_{0}^{2}\left[\frac{1}{8} \sum_{k \neq l \neq m} \frac{P_{k l m}^{(r)}}{P_{s}^{(r)}}+\frac{1}{4} \sum_{k \neq l \neq m=s} \frac{P_{k l s}^{(r)}}{P_{s}^{(r)}}+\frac{1}{4} \sum_{k=l \neq m} \frac{P_{k k m}^{(r)}}{P_{s}^{(r)}}\right] \int_{-\frac{1}{2 T}}^{\frac{1}{2 T}} \int_{\mid l}^{2 T \cos \pi f T \mid d f}
\end{aligned}
$$

$\sigma_{F W M-s i g n a l}^{2}=\frac{4}{\pi}\left[2\left(P_{s}^{(r)}\right)^{2}[x(0)]^{2} R_{0}^{2}\left[\frac{1}{8} \sum_{k \neq l \neq m} \frac{P_{k l m}^{(r)}}{P_{s}^{(r)}}+\frac{1}{4} \sum_{k \neq l \neq m=s} \frac{P_{k l s}^{(r)}}{P_{S}^{(r)}}+\frac{1}{4} \sum_{k=l \neq m} \frac{P_{k k m}^{(r)}}{P_{s}^{(r)}}\right]\right]$

\section{b. With Amplifier}

The schematic of the DB system for this case is as shown in Fig. 4. To simplify our analysis, and also to have a fair comparison with the MFs discussed before, we assume the ASE noise to be real.

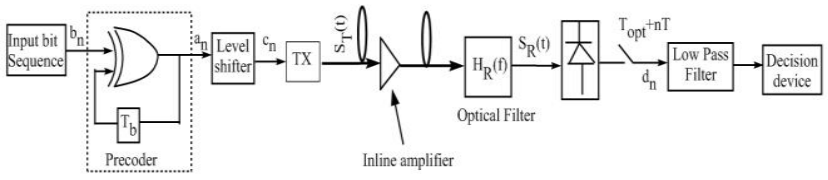

Fig. 4. Schematic of Duo-binary system with amplifier.

The received signal at receiver in this case will be given by $S_{R}(t)=\left\{\sqrt{2 P_{s}^{(r)}} \sum_{n} c_{n} u_{T}(t-n T)+\sum_{k=-M}^{M} \sqrt{2 S_{s p} \delta v} e^{\left.j(2 \pi k \delta v) t+\phi_{k}\right)}+\sqrt{2 P_{k l m}^{(r)}} e^{-j \theta_{k m m}}\right\} e^{j 2 \pi f c_{c} t}$

where $\phi_{k}$ is a random phase for each component of the spontaneous emission. In (56), first term is the signal component and the second and third terms represent ASE noise and FWM components respectively. Also, in (56), $P_{s p}=S_{s p} \cdot B_{0}$ where $S_{s p}=N_{s p}(G-1) h v$ and $M=\frac{B_{0}}{2 \delta v}$.

If received signal satisfies the DB pulse constraint, complex envelope of filtered output optical signal can be expressed as

$$
S_{R}\left(t_{\text {opt }}\right)=\left[\sqrt{2 P_{s}^{(r)}} d_{n} x(0)+n_{A S E}(t)+n_{F W M}(t)\right]
$$

The detected signal at the optimum sampling time is given as [17]

$$
i_{D}(t)=R_{0}\left(\sqrt{2 P_{S}^{(r)}} d_{n} x(0)+n_{A S E}(t)+n_{F W M}(t)\right)^{2}
$$

Assuming a high SNR, $P_{s}^{(r)}>>P_{F W M}^{(r)}$ and $P_{s}^{(r)}>>n_{A S E}^{2}(t)$, thus $P_{F W M}^{(r)}$ and $n_{A S E}^{2}(t)$ in (57) can be neglected. After photo detection, electrical signal at the optimum sampling instant is given as [17]

$$
\begin{aligned}
& i_{D}\left(t_{\text {opt }}\right)=R_{0} P_{S}^{(r)} d_{n}[x(0)]^{2}+n_{A S E-\text { signal }}\left(t_{\text {opt }}\right)+n_{F W M-A S E}\left(t_{\text {opt }}\right)+ \\
& n_{F W M-\text { signal }}\left(t_{\text {opt }}\right)+n_{\text {sh }}(t)+n_{\text {Th }}(t)
\end{aligned}
$$

where

$n_{A S E-\text { signal }}\left(t_{\text {opt }}\right), n_{F W M-A S E}\left(t_{\text {opt }}\right), n_{F W M-\text { signal }}\left(t_{\text {opt }}\right)$

represent the ASE-signal, FWM-ASE and FWM-signal beat noise. In (57), the second, the third and the fourth terms have zero mean and the variances are given as [17]

$$
\begin{gathered}
\sigma_{A S E-\text { signal }}^{2}=\frac{4}{\pi}\left[4 R_{0}{ }^{2} P_{s}^{(r)} x(0) S_{s p} B_{e}\right] \\
\sigma_{F W M-A S E}^{2}=\frac{4}{\pi}\left\{4 R_{0}^{2}\left[2 P_{s}^{(r)} x(0)\left[\frac{1}{8} \sum_{k \neq l \neq m} \frac{P_{k l m}^{(r)}}{P_{s}^{(r)}}+\frac{1}{4} \sum_{k \neq l \neq m=s} \frac{P_{k s}^{(r)}}{P_{s}^{(r)}}+\frac{1}{4} \sum_{k=l \neq m} \frac{P_{k m}^{(r)}}{P_{s}^{(r)}}\right]\right] \cdot S_{s p} B_{e}\right\} \\
\sigma_{F W M-s i g n a l}^{2}=\frac{4}{\pi}\left[2\left(P_{s}^{(r)}\right)^{2}[x(0)]^{2} R_{0}^{2}\left[\frac{1}{8} \sum_{k \neq l \neq m} \frac{P_{k l m}^{(r)}}{P_{s}^{(r)}}+\frac{1}{4} \sum_{k \neq l \neq m=s} \frac{P_{k l s}^{(r)}}{P_{s}^{(r)}}+\frac{1}{4} \sum_{k=l \neq m} \frac{P_{k k m}^{(r)}}{P_{s}^{(r)}}\right]\right]
\end{gathered}
$$

The $P_{e}$ is given as

$$
\begin{gathered}
P_{e}=P\left(\left|i_{D}\left(t_{\text {opt }}\right)\right|>R_{0} P_{s}^{(r)}[x(0)]^{2} / d_{n}=0\right) \\
P_{e}=\operatorname{erfc}\left(\frac{Q}{\sqrt{2}}\right)
\end{gathered}
$$

where 


$$
Q=\frac{R_{0} P_{s}^{(r)}[x(0)]^{2}}{\sigma_{n}}
$$

and

$\sigma_{n}^{2}=\sigma_{s h}^{2}+\sigma_{T h}^{2}+\sigma_{A S E-\text { signal }}^{2}+\sigma_{F W M-A S E}^{2}+\sigma_{F W M-\text { signal }}^{2}$

\section{RESULTS AND DISCUSSIONS}

For simulations, we used MATLAB and the commercial package Rsoft OptSim ${ }^{\mathrm{TM}}$ simulation software (Version 4.0) that gave us an environment almost like exact physical realization of a WDM system. Through the aforementioned we are able to validate the proposed model simulated on MATLAB (referred to as 'Theoretical') with the results simulated on OptSim (referred to as 'Simulation'). The essential components to build the WDM link architecture shown in Fig. 1 is as detailed in [14], assignment of the central wavelength of the channel is based on the ITU-T recommendation G.694.1 [15], and the configuration and various parameter values of the transmitters, receivers, filters, SSMF and DCF are as detailed in [16]. The required BER value is set to $10^{-12}$ (i.e., $Q$-factor value of 7 or $16.9 \mathrm{~dB}$ on the electrical scale) with BER ( $Q$-factor) values being evaluated for the most distorted channel; the amplifier used has a fixed output power level of $10 \mathrm{dBm}$ and the other physical layer parameters are identical as in $[11,12,14,16]$.

From Fig. 5 and 6 it can be observed that the $Q$-factor values degrade with the introduction of the amplifier owing to the ASE noise. Also, it can be seen that compared to when

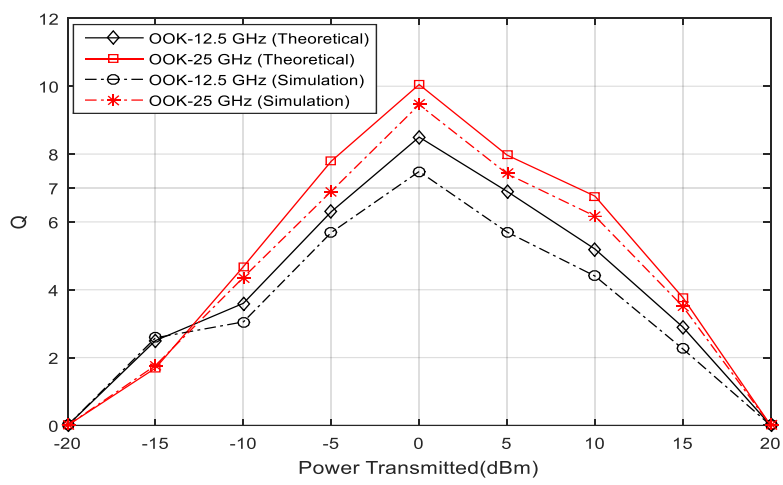

(a) the channel spacing is set to $12.5 \mathrm{GHz}$; better $Q$-factor performance is obtained when the channel spacing is fixed to $25 \mathrm{GHz}$. However, in both the channel spacing value cases, DB MF outperforms the DPSK and OOK MFs as it is more resilient to various considered PLIs. Overall, in all the cases, with increase in transmitted power, the $Q$-factor increases and reaches a maximum at $0 \mathrm{dBm}$ since PLI effects are low at lower transmitted powers; however, as transmitted power is increased beyond $0 \mathrm{dBm}$, the $Q$-factor values start to decrease since the wavelengths begin to overlap and the PLIs start to dominate.

Another important point to note from Fig. 5 and 6 is that the results obtained via OptSim provide degraded values of $Q$-factor in comparison to the exact theoretical approach. This occurs since OptSim simulates the non-linear fiber using the Split Step Fourier or Time Domain Split Step technique which provides an approximate solution to the Nonlinear Schrodinger equation (NLSE) that defines pulse evolution inside a SSMF [14]. However, even with minor deviation(s) in the $Q$-factor values, Fig. 5 and 6 shows that the simulation results follow the theoretical result suggesting that the proposed MLR model accurately calculates $Q$-factor for the considered MFs.

In order to evaluate the variation of $Q$-factor with channel spacing, we varied transmitted powers as $-5,0$, and $+5 \mathrm{dBm}$, and also considered deployment of an amplifier. Again, results (see Fig. 7) are found for worst affected (central) channel. From the figure, it can be observed that (i) in all three

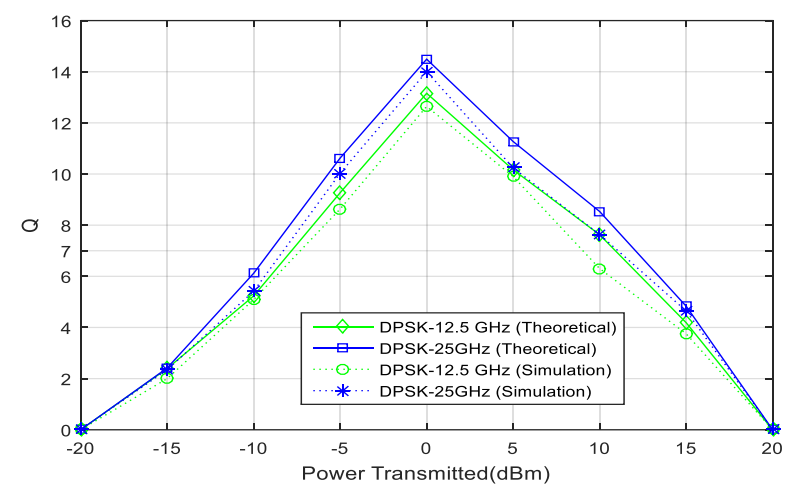

(b)

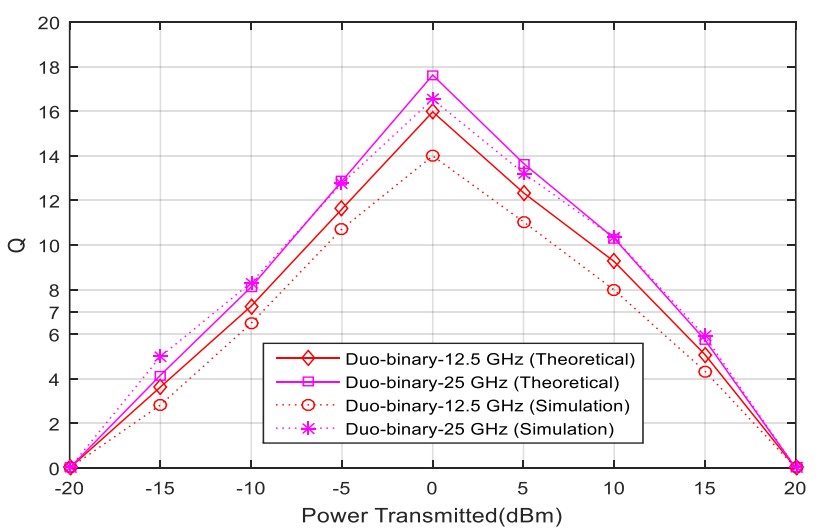

(c)

Fig.5. Variation of $Q$-factor with transmitted power for channel separation of $25 \mathrm{GHz}$ and $12.5 \mathrm{GHz}$, without amplifier (i.e. $L=80 \mathrm{kms}$ ) for the worst affected (central) channel as (a) OOK, (b) DPSK, and (c) DB. 


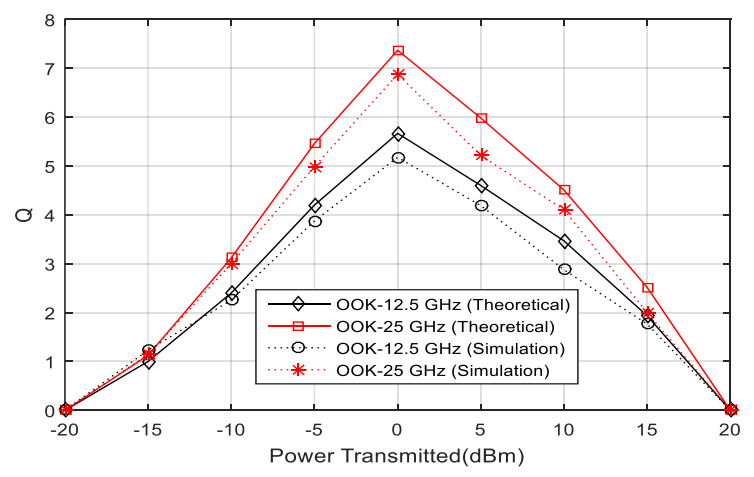

(a)

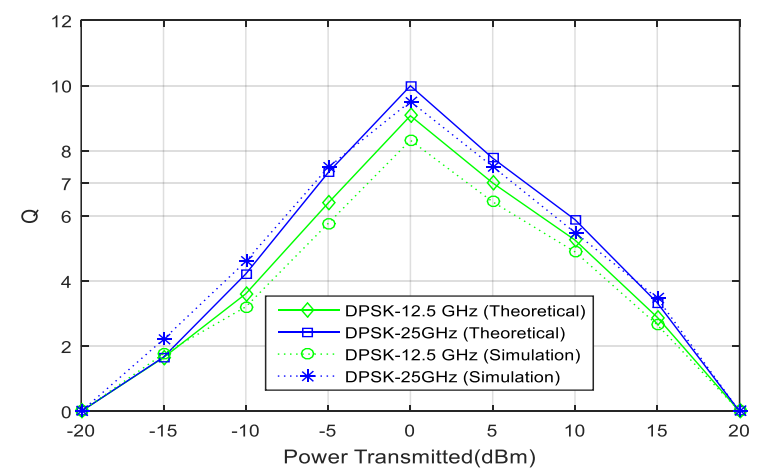

(b)

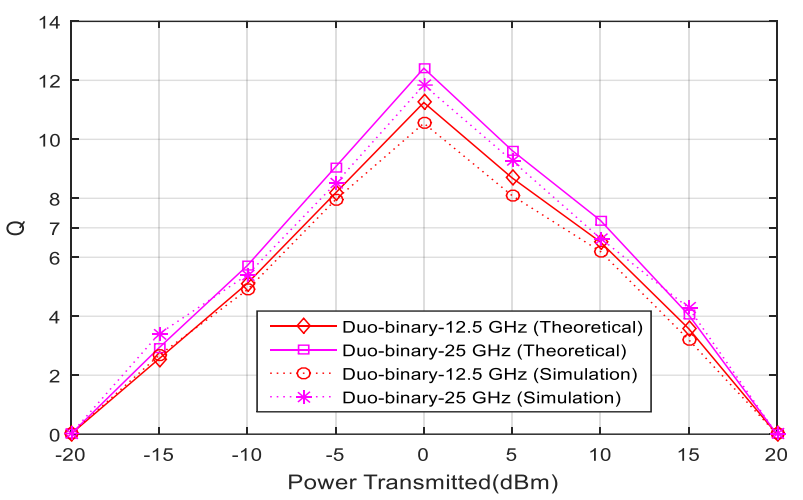

(c)

Fig.6. Variation of $Q$-factor with transmitted power for channel separation of $25 \mathrm{GHz}$ and $12.5 \mathrm{GHz}$, with amplifier (i.e. $L=120 \mathrm{kms}$ ) for the worst affected (central) channel as (a) OOK, (b) DPSK, and (c) DB.

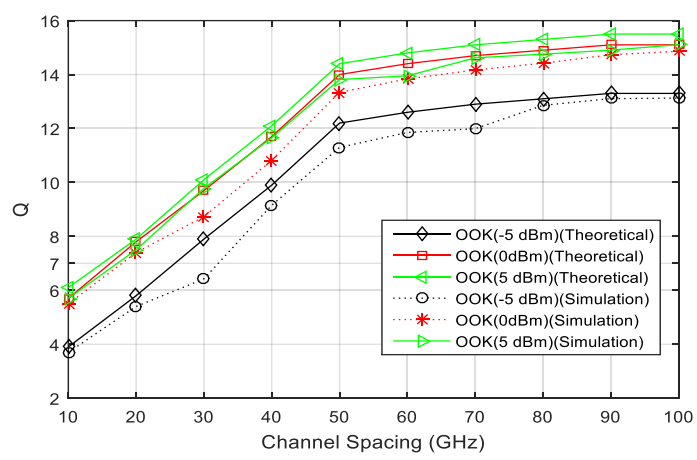

(a)

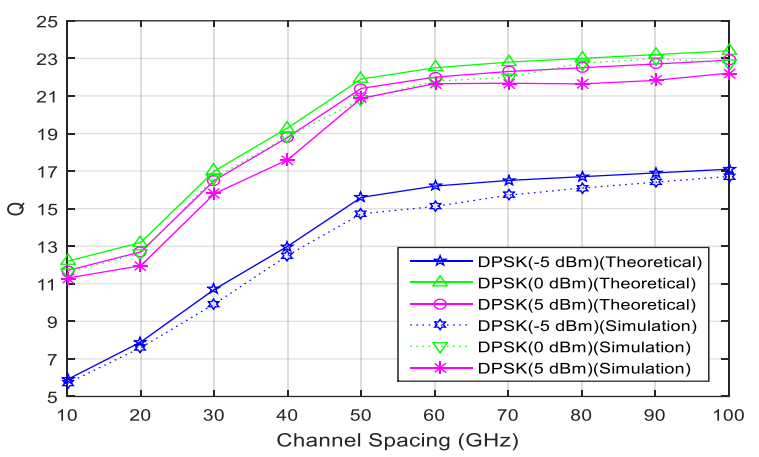

(b)

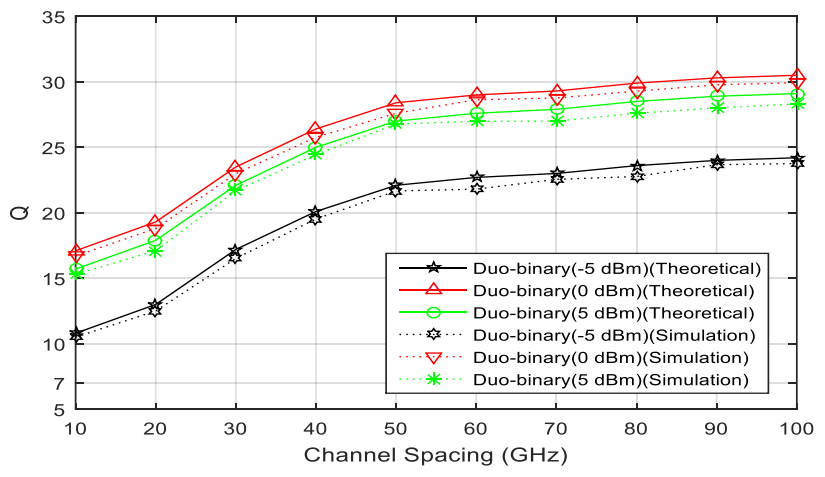

(c)

Fig.7. Variation of $Q$-factor with channel spacing for various transmitted power values for worst affected (central) channel as (a) OOK, (b) DPSK, and (c) DB. 
Table II Minimum channel spacing values required to achieve $\mathrm{Q}=7$ by the various considered modulation formats.

\begin{tabular}{|c|c|c|c|c|}
\hline & Transmitted Power & $-5 \mathrm{dBm}$ & $0 \mathrm{dBm}$ & $+5 \mathrm{dBm}$ \\
\hline Modulation Format & \multirow{4}{*}{ 胥 } & & & \\
\hline OOK & & 25.7 & 16.1 & 15.0 \\
\hline DPSK & & 15.5 & 2.4 & 1.7 \\
\hline DB & & 2.6 & 1.3 & 0.6 \\
\hline
\end{tabular}

Table III Spectral Efficiency achieved by the various considered modulation formats for different transmitted powers.

\begin{tabular}{|c|c|c|c|c|}
\hline Modulation Format & Transmitted Power & $-5 \mathrm{dBm}$ & $0 \mathrm{dBm}$ & $+5 \mathrm{dBm}$ \\
\hline OOK & \multirow{3}{*}{ 苞 } & 1.556 & 2.4844 & 2.666 \\
\hline DPSK & & 2.580 & 16.666 & 23.529 \\
\hline$\overline{\mathrm{DB}}$ & & 15.384 & 30.769 & 66.666 \\
\hline
\end{tabular}

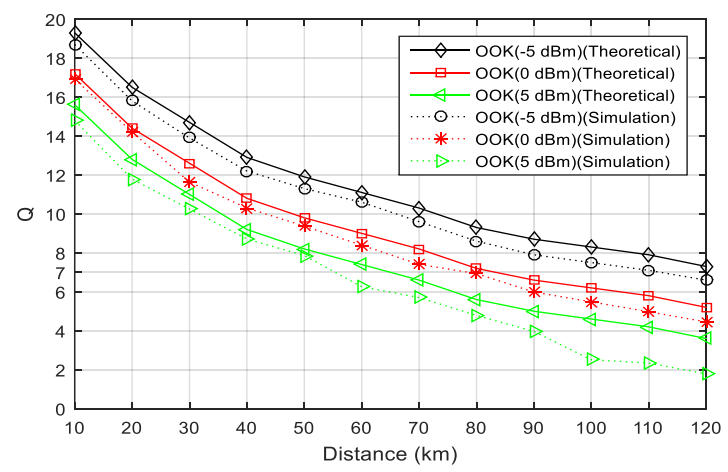

(a)

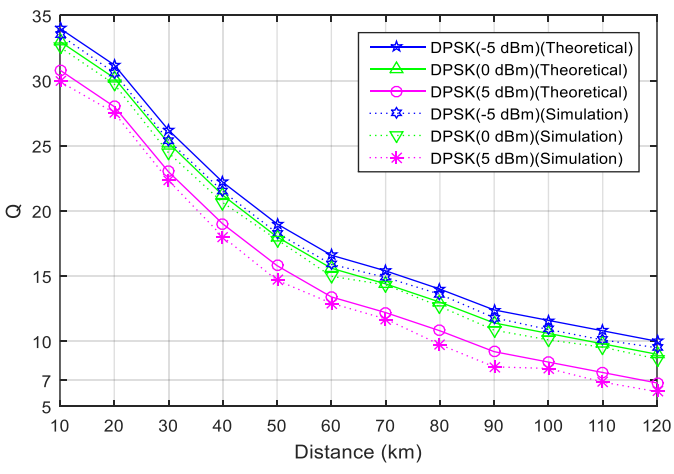

(b)

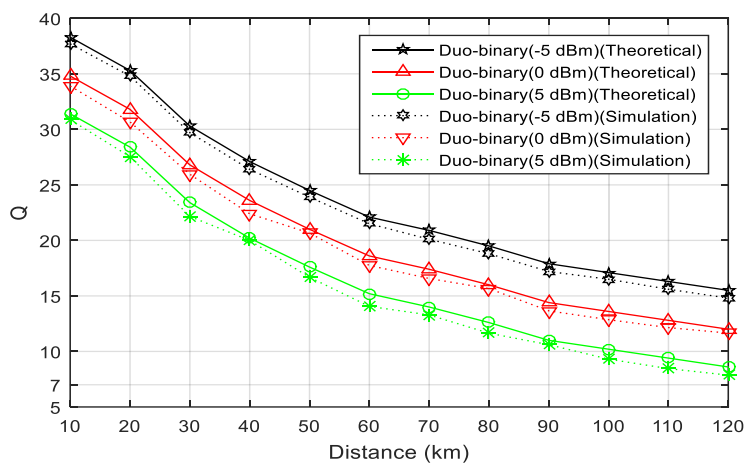

(c)

Fig.8. Variation of $Q$-factor with distance for various values of transmitted powers for the worst affected (central) channel as (a) OOK, (b) DPSK, (c) and DB.

MFs, for given channel spacing, performance improves with increase in transmitted power, (ii) for given channel spacing, performance (in terms of $Q$-factor) of $\mathrm{DB}$ is better than OOK and DPSK, and (iii) if MF is OOK or DPSK, with transmitted power of $-5 \mathrm{~dB}$ and channel spacing of $10 \mathrm{GHz}$, it is not possible to achieve $Q=7$, whereas the same is possible in the case of the DB MF.

For the considered MFs, the minimum channel spacing values in order to achieve a $Q$-factor value of 7 , and the corresponding spectral efficiency(s) are shown in Table II and III, respectively.

Finally, in order to observe the performance of various MFs with variation in distance, for the worst affected channel, we plotted the $Q$-factor with distance for various values of transmitted powers and set the channel spacing to $12.5 \mathrm{GHz}$. It can be seen from Fig. 8 that with increase in both, the distance and the transmitted power, the $Q$-factor decreases and OOK shows the worst performance whereas, DB maintains the best performance since it better alleviates the effect of all the PLIs. Also, for the shorter distances, the DPSK and the DB MFs show similar performance; however, for longer distances, DB MF outperforms DPSK as it is much more resilient to all the PLIs.

Overall, from all the results it can be inferred that an increase in the number of users is possible provided (i) the bandwidth is increased simultaneously by setting the same channel spacing, or (ii) the channel spacing can be decreased simultaneously by keeping the bandwidth as narrow as 
possible. Hence, each MF has its own advantage(s) and /or disadvantage(s), as the service providers are usually interested in both, narrowing the bandwidth and reducing the crosstalk between the channels. Also, the reduction in the channel spacing implies an imposition of stricter requirements on the system characteristics; however, it leads to stable system characteristics.

\section{V.CONCLUSION}

In this work we have compared the performance of a 40Gbps MLR WDM network based on the (i) NRZ-OOK, (ii) DPSK, and (iii) Duo-binary modulation formats, with the variation of power and in the presence of various PLIs. In view of the aforementioned, we have mathematically detailed the MLR network considering various modulation formats, and have also validated the proposed theoretical model's results by comparing them with those obtained through simulations from OptSim, which to our knowledge has not been done in any existing study thus far. Our simulation results show that the Duo-binary modulation format is perfectly suitable for high spectral-efficient MLR systems owing to its high resistance to the various PLIs.

\section{REFERENCES}

[1] A. Nag, M. Tornatore, B. Mukherjee, "Optical network design with mixed line rates and multiple modulation formats", IEEE Journal of Lightwave Technology, vol. 28, no. 4, pp. 466-475, 2010.

[2] M. Batayneh, D.A. Schupke M. Hoffmann, A. Kirstaedter, B. Mukherjee, "On routing and transmission-range determination of multibit-rate signals over mixed-line-rate WDM optical networks for carrier Ethernet", IEEE/OSA Transactions on Networking, vol. 19, no. 5, pp. 1304-1316, 2011.

[3] R. Ramaswami, K.N. Sivarajan, G.H. Sasaki, "Optical Networks: A Practical Perspective", Elsevier, 2010.

[4] S.P. Singh, S. Sengar, R. Bajpai, S. Iyer, "Next-Generation Variable-Line-Rate Optical WDM Networks: Issues and Challenges", Journal of Optical Communications, De Gruyter, vol. 34, no. 1, pp. 331-350, 2013..

[5] M. Haris, "Advanced Modulation Formats for High-Bit Rate Optical Networks", Ph.D. Dissertation, Georgia Institute of Technology, 2008.

[6] P.J. Winzer, R.J. Essiambre, "Advanced optical modulation formats", Proceedings of the IEEE, vol. 94, no. 5, pp. 952985, 2006

[7] S. Zhang, "Advanced Optical Modulation Formats in highspeed Lightwave system". Ph.D. Dissertation, University of Kansas, 2008.

[8] P. Winterling, "Optical Modulation Methods: For HighSpeed Networks and the Consequences for Test Equipment. JDSU", White Paper, 2008.

[9] M. Joindot, G. Bosco, A. Carena, V. Curri, P. Poggiolini, "Fundamental performance limits of optical duobinary", Optics Express, vol. 16, no. 24, pp. , 2008

[10] G. Bosco, A. Carena, V. Curri, R. Gaudino, P. Poggiolini, "Quantum Limit of Direct-Detection Receivers Using Duobinary Transmission", IEEE Photonics Technology Letters, vol. 15, no. 1, pp. 102-104, 2003.

[11] S.P. Singh, S. Kar, V.K. Jain, "Effect of four wave mixing on optimal placement of optical amplifier in WDM star networks", Fiber and Integrated Optics, vol. 25, no. 2, pp.
111-140, 2006.

[12] A.V.T Cartaxo, "XPM in IM/DD WDM system with multiple optical amplifiers and dispersion compensators", IEEE Journal of Lightwave Technology, vol. 17, no. 2, pp. 178-190, 1999.

[13] S. Iyer, S.P. Singh, "Theoretical Evaluation of combined nonlinearities and ASE noise Penalties in Optical Star WDM Networks Based on ITUT conforming Optical Fibers", IETE Journal of Research, vol. 58, no. 6, pp. 482-92, 2012.

[14] S. Iyer, S.P. Singh, "Impact of Combined Non-Linearities and ASE Noise on Performance of 10 Gbps All Optical Star WDM Networks", Scientific Research, Communications and Network, vol. 3, no. 4, pp. 235-249, 2011.

[15] ITU-T Recommendation G.694.1. Spectral grids for WDM applications: DWDM frequency grid- Series G: Transmission Systems and Media, Digital Systems and Networks. 2012.

[16] S. Iyer, S.P. Singh, "Spectral and Power-Efficiency Investigation in Single and Multi-Line-Rate Optical Wavelength Division Multiplexed (WDM) Networks", Photonic Network Communication, Springer, vol. 33, no. 1, pp. 39-51, 2017.

[17] S. Iyer, S. Sengar, R. Bajpai, S.P. Singh," On the Performance of MLR Optical WDM Network Based on ITU-T Conforming Fibers in the Presence of Dominant Physical Layer Impairments", Journal of Communications and Information Networks, vol. 3, no. 2, pp. 23-40, 2018. 